\title{
Percepção do Apoio Social e Configuração Sintomática na Anorexia Nervosa ${ }^{1}$
}

\author{
Carolina Leonidas ${ }^{1}$ \\ ${ }^{1}$ Universidade Federal do Triângulo Mineiro, MG, Brasil.
}

\author{
Manoel Antônio dos Santos ${ }^{2}$ \\ ${ }^{2}$ Universidade de São Paulo, SP, Brasil.
}

Resumo: O estudo investigou a configuração da rede social significativa de uma mulher com anorexia nervosa, buscando articular a percepção do apoio social recebido e o curso do transtorno. Utilizou-se como estratégia metodológica o estudo de caso e o enfoque das redes sociais para análise dos dados. Participou do estudo uma jovem de 24 anos com diagnóstico de anorexia nervosa do tipo compulsivo-purgativo. Os instrumentos utilizados foram: roteiro de entrevista semiestruturada, Genograma, Mapa de Rede e consulta ao prontuário hospitalar. O conteúdo da entrevista foi transcrito na íntegra e literalmente, e o material coligido constituiu o corpus da pesquisa. O Genograma e o Mapa de Rede foram analisados de acordo com as recomendações da literatura e o referencial teórico utilizado. Os resultados apontaram dificuldades no estabelecimento de vínculos e sofrimento decorrente da percepção de falta de suporte da rede familiar. Certos aspectos da dinâmica de personalidade da participante resultavam em afastamento das pessoas e consequente isolamento social, o que restringe $o$ potencial de apoio da rede e suscita vivências de solidão e desamparo. Enfatiza-se a necessidade de aproveitar o potencial das redes de apoio na assistência em saúde para fortalecer os laços sociais de jovens com diagnóstico de anorexia.

Palavras-chave: Anorexia Nervosa, Redes Sociais, Apoio Social, Caso Clínico.

\section{Perception of Social Support and Symptomatic Configuration in Anorexia Nervosa}

\begin{abstract}
This study investigated the configuration of the significant social network of a 24-yearold girl diagnosed with Compulsive-Purgative AN (AN) to articulate the participant's perception of social support and the symptoms of her eating disorder. The methodological strategy involved a case study and the social networks approach for data analysis. Semi-structured interview, Genogram, Network Mapping and consultations to hospital records were used as instruments. The content of the interviews was transcribed in full and the collected material constituted the research corpus. The genogram and network mapping were analyzed according to recommendations from the scientific literature and to the theoretical framework used. The results showed issues in establishing emotional bonds and suffering due to perceived lack of social support from the family network. In addition, aspects of the participant's personality dynamics resulted in emotional withdrawal and consequent social isolation, restricting the potential support of the network and leading to experiences of loneliness and helplessness. We emphasize the need for integration of social networks in healthcare in order to strengthen the social bonds of young women diagnosed with anorexia.
\end{abstract}

Keywords: Anorexia Nervosa, Social Networks, Social Support, Clinical Case.

${ }^{1}$ Este estudo recebeu apoio financeiro da Fundação de Amparo à Pesquisa do Estado de São Paulo (FAPESP), por meio da concessão de bolsa de Mestrado à primeira autora, processo no 2010/1257-7, sob orientação do segundo autor. 


\title{
Percepción del Apoyo Social y Configuración Sintomática de la Anorexia Nerviosa
}

\begin{abstract}
Resumen: El estudio investigó la configuración de la red social significativa de una mujer con anorexia nerviosa buscando articular la percepción del apoyo social recibido con el curso del trastorno. La estrategia metodológica utilizada fue el estudio de caso y el enfoque en las redes sociales aplicado en el análisis de datos. Una joven de 24 años con diagnóstico de anorexia nerviosa del tipo compulsivo-purgativo participó en el estudio. Los instrumentos utilizados fueron: entrevista semiestructurada, genograma, mapa de red y consultas a los registros hospitalarios. El contenido de la entrevista fue transcrito completo y literalmente, y el material recopilado compuso el corpus de la investigación. El genograma y el mapa de red se analizaron de acuerdo con las recomendaciones de la literatura científica y el marco teórico utilizado. Se percibieron dificultades en el establecimiento de vínculos y sufrimientos que resultan de la percepción de falta de apoyo de la red familiar. Además, determinados aspectos de la dinámica de la personalidad de la participante llevaron a la retirada de las personas y el consiguiente aislamiento social, lo que restringe el potencial de apoyo de la red y conduce a experiencias de soledad e impotencia. Se enfatiza la necesidad de aprovechar el potencial de las redes de apoyo en la asistencia sanitaria para fortalecer los lazos sociales de mujeres jóvenes diagnosticadas con anorexia.
\end{abstract}

Palabras clave: Anorexia Nerviosa, Redes Sociales, Apoyo Social, Caso Clínico.

\section{Introdução}

Nos dias atuais tem sido conferida importância crescente aos transtornos alimentares (TAs). Esses quadros psicopatológicos são marcados por graves perturbações no comportamento alimentar, acompanhadas de intenso sofrimento psíquico e ameaça à integridade física (American Psychiatric Association, 2013; Leonidas \& Santos, 2012). Tais transtornos podem assumir um curso crônico e incapacitante, resultando em inúmeros prejuízos físicos e psicológicos para o indivíduo acometido (Eddy et al., 2017). Adolescentes do sexo feminino, de todas as camadas sociais, são os grupos populacionais mais suscetíveis ao desenvolvimento desses quadros (Leonidas \& Santos, 2020; Valdanha, Scorsolini-Comin, \& Santos, 2013).

Essa problemática é de interesse para a Psicologia, uma vez que a incidência e prevalência de TAs vêm aumentando desde a segunda metade do século XX (Leonidas, Nazar, Munguía, \& Santos, 2019), especialmente entre mulheres (Smink, Hoeken, \& Hoek, 2013). Concomitantemente às preocupantes taxas de prevalência, também houve incremento no número de publicações dedicadas ao tema, que investigam tanto os fatores culturais e sociais - dentre os quais se destaca a associação entre atratividade física e magreza na sociedade contemporânea - quanto os fatores relacionados à influência da família, da personalidade e dos aspectos somáticos (King, Gerish, \& Schreiber, 2019).

Por se tratar de um quadro mobilizador de intensa carga emocional, a incidência dos TAs tem impacto extremamente negativo na qualidade de vida do indivíduo acometido (Oliveira-Cardoso, Von Zuben, \& Santos, 2014) e nas relações familiares, abalando a dinâmica familiar (Leonidas et al., 2019; Siqueira, Santos, \& Leonidas, 2020). Apesar de ainda não existir um consenso a respeito da origem desses quadros, reconhece-se que a etiologia envolve a interação dinâmica de vários fatores, tanto intrínsecos quanto extrínsecos ao indivíduo (King, Gerish \& Schreiber, 2019; Leonidas \& Santos, 2020; OliveiraCardoso \& Santos, 2012). Tais fatores combinados dão início, precipitam e/ou mantêm o transtorno, gerando alterações em vários aspectos da vida pessoal, dentre eles os relacionamentos interpessoais e afetivo-sexuais - uma vez que a vulnerabilidade emocional dificulta o estabelecimento e a preservação de vínculos (Leonidas \& Santos, 2015a).

Anorexia nervosa (AN) e bulimia nervosa (BN) são os tipos mais prevalentes de TAs, segundo o Manual Diagnóstico e Estatístico de Transtornos 
Mentais, 5a edição (DSM-5) (American Psychiatric Association, 2013). A primeira é caracterizada por uma recusa sistemática a manter o peso corporal na faixa normal mínima adequada à idade e altura, além de um temor intenso de ganhar peso, com distorção significativa da imagem corporal (American Psychiatric Association, 2013). Em termos psicológicos, as mulheres com quadro de AN vivenciam sentimentos de inferioridade, insegurança e inadequação, e apresentam traços de personalidade que abrangem perfeccionismo exagerado, obsessividade, retraimento social e condutas de evitação (Leonidas et al., 2019; Oliveira-Cardoso \& Santos, 2012; Valdanha, Scorsolini-Comin, \& Santos, 2013).

Já a pessoa com BN apresenta episódios recorrentes de compulsão alimentar, seguidos de comportamentos compensatórios inadequados, com claro objetivo de evitar o aumento de peso (American Psychiatric Association, 2013). Esse quadro psicopatológico remete a um apetite ávido, ao qual o indivíduo se entrega com voracidade e edacidade (Brusset, 2003). No entanto, essa voracidade não é direcionada apenas à alimentação, mas alcança uma série de objetos e atividades, tais como estudos, vida profissional e relações amorosas o que acaba resultando em emoções desadaptativas e relacionamentos caóticos (Leonidas \& Santos, 2017; Leonidas, Crepaldi, \& Santos, 2013). Essa categoria diagnóstica também está associada à apresentação de comportamentos impulsivos e comorbidades psiquiátricas, tais como traços histriônicos e transtorno de personalidade do tipo borderline (Leonidas et al., 2013; Rosa \& Santos, 2011). A presença de vários desses sintomas e traços de personalidade potencializa o risco à integridade física e psíquica.

As características de personalidade que marcam os indivíduos acometidos por TAs geralmente dificultam o fornecimento de apoio social por parte das pessoas que compõem suas redes sociais significativas (Limbert, 2010). Essas redes podem ser definidas como as relações interpessoais que os indivíduos estabelecem ao longo da vida, tanto na família quanto no plano macrossocial (Lavall, Olschowsky, \& Kantorski, 2009; Moré, 2005; Moré \& Crepaldi, 2012). Tais relações configuram a rede social pessoal de cada sujeito, que corresponde à "soma de todas as relações que um indivíduo percebe como significativas ou define como diferenciadas da massa anônima da sociedade" (Sluzki, 1997, p. 42).

Desde o nascimento, o indivíduo é inserido em uma história que o antecede e que funciona como uma pauta familiar, na qual ele irá se inserir e desenvolver sua própria configuração psíquica. Essa inscrição será sempre singular, porém estabelecida a partir de elementos preexistentes na trama de cada rede familiar (Attili, Di Pentima, Toni, \& Roazzi, 2018). A isso se segue a formação de inúmeros tipos de vínculos, desde uma interação regular e íntima com membros do contexto micro e macrossocial em que a pessoa vive até relações esporádicas e distantes (Sluzki, 1997). Essas interações estabelecidas pelo indivíduo, no marco das relações familiares, sociais e pessoais, constituem o processo incessante de formação da identidade. Dessa forma, todas as pessoas que fazem parte da rede social significativa colaboram de forma muito particular para a construção da personalidade do sujeito e para modular seu funcionamento.

A despeito dos TAs terem adquirido notória visibilidade nos últimos anos, muitas características da evolução e do tratamento desses quadros ainda permanecem desconhecidas (Souza, Valdanha-Ornelas, Santos, \& Pessa, 2019), o que contribui para perpetuar a incompreensão e os pré-julgamentos por parte dos familiares, parceiros afetivos, amigos, professores e colegas de trabalho. Esse desconhecimento incrementa o isolamento social das pessoas acometidas, intensificando seus sentimentos de solidão e desamparo frente aos desafios encontrados no processo adaptativo, o que resulta em maior sofrimento (Goulart \& Santos, 2012; Leonidas \& Santos, 2015a, 2015b). Por essa razão, ao planejar o cuidado a ser dispensado a esses pacientes, é fundamental que os profissionais de saúde conheçam suas redes sociais e o apoio percebido e recebido.

Levando-se em consideração que as relações interpessoais desempenham um papel crucial no enfrentamento de doenças estigmatizantes (Lavall, Olschowsky, \& Kantorski, 2009), parte-se da hipótese de que o potencial do apoio social percebido pelo indivíduo acometido por TA encontra-se prejudicado, uma vez que os aspectos constitutivos de sua personalidade podem comprometer suas relações interpessoais, familiares e afetivas. Adolescentes com AN apresentam taxas de comorbidade psiquiátrica elevadas (Trainor et al., 2020), de modo que o funcionamento psicológico é consideravelmente prejudicado, gerando dificuldades na formação e manutenção de vínculos. Como consequência do enfraquecimento do potencial de apoio social da rede, pode ocorrer agravamento dos sintomas, com incremento do sofrimento 
e de sentimentos de desamparo e menos-valia, culminando na cronicidade do quadro psicopatológico (Leonidas \& Santos, 2014).

A literatura sobre o apoio social percebido no contexto dos TAs é escassa, o que dificulta a consideração dessa dimensão no plano de tratamento. A partir desses pressupostos, este estudo teve por objetivo investigar a configuração da rede social significativa de uma mulher com AN, buscando elucidar possíveis articulações entre a percepção do apoio social recebido por meio da rede e o curso do TA.

\section{Método}

\section{Delineamento do estudo}

Esta pesquisa se configura como um estudo exploratório de corte transversal. Adotou-se como delineamento metodológico o estudo de caso, um enfoque qualitativo de pesquisa. Essa estratégia foi considerada pertinente tendo em vista o caráter exploratório do estudo e a necessidade de elucidar aspectos da rede social na perspectiva da pessoa que vivencia a condição do TA. Dentre as abordagens existentes, optou-se pela proposta por Stake (2005), enriquecida com as contribuições de Peres e Santos (2005), haja vista que esse enfoque parece ser o que mais se ajusta à questão de pesquisa que norteia este estudo. Considera-se que o caso é um sistema delimitado, podendo ser uma pessoa, um grupo ou mesmo uma comunidade. Nas várias definições conceituais existentes sobre a estratégia de estudo de caso, há uma ideia recorrente de que esse enfoque não se refere apenas a um método, mas também à escolha de um determinado objeto a ser investigado (Godoy, 2006). A essência de um estudo de caso é a delimitação do fenômeno estudado. Neste estudo, circunscreveu-se como fenômeno a ser investigado a configuração da rede social significativa de uma mulher com anorexia e o modo como a percepção do apoio social recebido dessa rede articula-se com os sintomas de TA.

\section{Aspectos éticos}

O estudo foi desenvolvido junto ao Grupo de Assistência em Transtornos Alimentares (Grata), vinculado ao ambulatório de Nutrologia do Hospital das Clínicas da Faculdade de Medicina de Ribeirão Preto, Universidade de São Paulo (Oliveira-Cardoso et al., 2018; Souza et al., 2019). O projeto foi aprovado pelo Comitê de Ética em Pesquisa da instituição (processo $n^{\circ} 2155 / 2010$ ). Além disso, a participante firmou o Termo de Consentimento Livre e Esclarecido (TCLE). Para preservar o anonimato e a confidencialidade dos dados, na apresentação do caso clínico os nomes próprios foram substituídos por nomes fictícios.

\section{Descrição da participante}

Este estudo consiste em um recorte da dissertação de mestrado intitulada Redes sociais e apoio social no contexto dos transtornos alimentares (Leonidas, 2012), da qual participaram 12 pacientes com diagnóstico de TAs que se encontravam em atendimento regular no serviço interdisciplinar de um hospital universitário do interior paulista. Dessa amostra consecutiva, que correspondia ao total de pacientes atendidas no período de seis meses que preencheram os critérios de inclusão do estudo, foi extraído o caso de uma participante de 24 anos, com diagnóstico de AN do tipo compulsivo-purgativo. Os critérios de seleção foram: a) estar em regime de tratamento ambulatorial; b) apresentar discurso coerente e orientado no tempo e espaço; c) apresentar características que configuram um "caso", de acordo com a definição da literatura (Stake, 2005). A participante foi selecionada por proporcionar uma visão abrangente acerca dos modos como o suporte social percebido como proveniente da rede de apoio se articula com a apresentação dos sintomas.

\section{Procedimento de coleta e análise dos dados}

Para a coleta de dados foram utilizados: roteiro de entrevista semiestruturada, Genograma, Mapa de Rede e consulta ao prontuário hospitalar. A aplicação dos instrumentos foi realizada individualmente, em sala reservada da instituição hospitalar. A entrevista teve duração aproximada de 60 minutos e foi gravada em áudio mediante anuência da participante. A dinâmica da entrevista envolveu também a construção do Genograma e do Mapa de Rede. Posteriormente, o conteúdo gravado foi transcrito na íntegra e literalmente, e o material coligido constituiu o corpus de pesquisa.

De forma concomitante à análise da entrevista, o Genograma e o Mapa de Rede - construídos à mão pela pesquisadora durante a etapa de coleta de dados - foram diagramados com uso do 
CorelDRAW Graphics Suite, um programa específico para criação de elementos gráficos que facilita a visualização dos resultados. O Genograma e o Mapa de Rede foram analisados de acordo com as recomendações e o enquadre teórico proposto por Sluzki (1997).

Para a triangulação dos dados foram percorridas duas etapas, de acordo com os passos metodológicos recomendados por Yin (2005). Na primeira, realizou-se uma descrição abrangente do caso, organizada de forma a destacar a maneira como a participante percebe o apoio recebido de sua rede social e como essa percepção se articula com os aspectos importantes de sua vida de relações e seus sintomas de TA. Na segunda, utilizou-se a técnica de "construção da explanação" (Yin, 2005), com objetivo de analisar exaustivamente os dados do caso investigado e construir uma explanação psicodinâmica sobre o fenômeno investigado.

Uma vez realizada a triangulação dos dados, a descrição abrangente do caso e a construção da explanação, deu-se a elaboração da narrativa clínica, que seguiu as recomendações propostas por Goulart e Santos (2015) e Kreling e Santos (2005) para articulação teórico-clínica do material.

\section{Resultados e discussão}

No momento da entrevista, Marcela encontrava-se em tratamento no serviço havia apenas um mês. No momento da admissão a participante apresentou os seguintes parâmetros antropométricos peso: $53,7 \mathrm{~kg}$, altura: $1,63 \mathrm{~m}$, resultando em um Índice de Massa Corporal (IMC) de $20,7 \mathrm{~kg} / \mathrm{m}^{2}$, considerado compatível com a faixa de normalidade. Assim, do ponto de vista da relação peso-altura, Marcela encontrava-se eutrófica, apesar dos sintomas de TA.

Segundo os registros extraídos do prontuário médico da participante, o diagnóstico era de AN, subtipo compulsivo-purgativo, em comorbidade com transtorno de personalidade do tipo borderline e episódio depressivo moderado. De acordo com o DSM-5 (American Psychiatric Association, 2013), a combinação da AN com outros transtornos psiquiátricos comórbidos indica padrões de intensa impulsividade, instabilidade e descontrole emocional, que se refletem nos relacionamentos interpessoais, na autoimagem e na organização dos afetos. O transtorno de personalidade se manifesta por graves dificuldades para lidar com a separação do outro - vivenciada como abandono, real ou imaginário -, já que essa vivência ou a percepção de rejeição pode acarretar sentimento de raiva inadequada e ações impulsivas, tais como comportamento autolesivo, desde automutilação até tentativa de suicídio (Rosa \& Santos, 2011). A paciente pode ainda apresentar reações de tristeza, ciúme e apego afetivo exacerbado, além de teimosia e insatisfação pessoal. Quando esses comportamentos e sentimentos se apresentam de forma recorrente, intensa e persistente, produzem um padrão existencial marcado por dificuldades de adaptação ao ambiente social e suscetibilidade ao transbordamento afetivo (Leonidas \& Santos, 2013; Kreling \& Santos, 2005), em decorrência de um déficit de controle racional.

Vale ressaltar que o diagnóstico de AN foi estabelecido pela equipe interdisciplinar considerando o retrospecto e a história psiquiátrica da paciente. Apesar de ter um IMC dentro da normalidade (American Psychiatric Association, 2013), Marcela apresentava quadros de restrição alimentar grave, alternados com momentos de compulsão alimentar (binge eating) seguidos de rituais purgativos. Além disso, já havia tido IMC bastante rebaixado em outros períodos de sua vida.

Antes de ser admitida no serviço, Marcela já havia se submetido a outros tratamentos para o TA. Relatou que, há cerca de 10 anos, quando tinha 14 anos, havia iniciado o quadro de restrição alimentar, acompanhado de uso de laxantes, diuréticos e atividade física intensa. Em poucos meses o quadro se agravou, o que a levou a uma internação prolongada de sete meses, quando chegou a pesar $29 \mathrm{~kg}$, com IMC $=10,1 \mathrm{~kg} / \mathrm{m}^{2}$, que caracteriza quadro gravíssimo de desnutrição, considerado incompatível com a vida. Após o período de hospitalização, logrou uma melhora discreta, mas, posteriormente, sua condição clínica permaneceu instável, alternando entre períodos de melhora e um quadro recorrente de piora. Essa instabilidade levou-a novamente a um estado de desnutrição muito grave, fazendo-a passar por mais três internações subsequentes, sendo uma delas em Hospital Dia, durante seis meses, intercalada com outras hospitalizações com intervalos mais curtos, de aproximadamente 15 dias. A participante relatou piora significativa dos sintomas alguns meses antes do início da busca de tratamento no serviço, com uso mais frequente de laxantes (cerca de 10 comprimidos por dia) e indução de vômitos com uma frequência de até três vezes por semana.

Na época em que a entrevista foi realizada, os dados antropométricos da participante não haviam 
sofrido mudanças significativas, em função do pouco tempo de tratamento que havia transcorrido desde a entrada no serviço até sua participação voluntária no estudo: seu peso era de $56,4 \mathrm{~kg}$ e seu IMC $=21,2 \mathrm{~kg} / \mathrm{m}^{2}$. Portanto nesse período houve recuperação do peso corporal. Contudo, as anotações psiquiátricas e nutricionais no prontuário indicavam que Marcela referia sentir-se muito cansada, pois se mantinha quase todo o tempo mergulhada no ciclo "comer, tomar laxante, vomitar". Embora houvesse reduzido o consumo diário de laxantes para seis comprimidos ao dia, não era sempre que conseguia manter tal controle de sua compulsão. Queixava-se frequentemente de fraqueza e tontura. Os vômitos aconteciam três vezes por semana, principalmente nos fins de semana, quando ingeria alimentos mais calóricos. Marcela contou que não gostava da imagem que via refletida no espelho e que sentia medo dos pais, pois eles não sabiam como lidar com seu problema.

Vale ressaltar que Marcela morava em um município distante de sua cidade de origem, onde cursava faculdade, mas voltava para a casa dos pais quase todos os finais de semana. A ocorrência maior dos vômitos coincide não apenas com a ingestão de alimentos mais calóricos como também com o retorno para a casa dos pais e o consequente contato mais próximo com a rede social familiar. A literatura especializada sugere que as famílias com um membro acometido por TA apresentam características de funcionamento bastante específicas, tais como relacionamentos familiares marcados por conflitos e turbulências, sentimentos de perda, culpa e sacrifício (Leonidas \& Santos, 2015a, 2015b; Siqueira et al., 2020). Uma pesquisa que investigou a percepção de adolescentes com AN e seus pais acerca do funcionamento familiar mostrou que mães e filhas têm visões diferentes em relação à dimensão da rigidez familiar (Laghi et al., 2017). Além disso, as filhas declararam estar menos satisfeitas com o ambiente familiar e classificaram suas famílias como menos comunicativas, menos flexíveis, menos coesas e mais desengajadas em comparação com adolescentes do grupo controle.

Outros estudos apontam que as relações que se desenvolvem nessas famílias são construídas sobre uma base frágil e vulnerável, com predominância de vínculos ambivalentes, dificuldades de comunicação da adolescente com os outros membros da família, triangulações, coalizações, expressão emocional empobrecida e intolerância em relação à separação e a reconhecer o espaço do outro (Costa \& Santos, 2016; Leonidas \& Santos, 2015a, 2015b; Oliveira-Cardoso et al., 2018). Nesse sentido, pode-se pensar nas dificuldades que Marcela apresenta com a alimentação, que são intensificadas durante sua estadia na casa dos pais, como possível via de expressão de sua dificuldade para lidar com os conflitos familiares e com os problemas que emergem em sua relação com o casal parental. Adicionalmente, é interessante notar que a mãe da participante era cozinheira e que a irmã mais velha era nutricionista, o que indica que duas pessoas da família com quem Marcela mantinha relacionamentos conflituosos também tinham a comida como um elemento organizador em suas vidas. Esse aspecto costuma ser recorrente em famílias de pessoas com TAs (Andrade \& Santos, 2009; Oliveira-Cardoso \& Santos, 2012).

O relacionamento de Marcela com os pais sempre foi bastante conflituoso, permeado por ameaças, cobranças e o que a jovem denomina de "chantagens emocionais". A participante caracterizou sua mãe como uma mulher com temperamento imprevisível e explosivo, sujeita a episódios de descontrole emocional. Em contrapartida, outros relatos indicam que Marcela mantém um relacionamento fusional e conflituoso com a figura materna, permeado por estreita dependência emocional e indiferenciação de limites eu-outro.

A participante definiu seu pai como uma pessoa calma, porém bastante crítica, com quem mantém um relacionamento vulnerável - ou seja, não há conflito explícito, mas existe a possibilidade da ocorrência de problemas sob condições adversas ou em fases de transição (Wendt \& Crepaldi, 2008). Nota-se que o padrão de relacionamento estabelecido com a figura paterna é emocionalmente distante, de maneira a evitar o contato com possíveis conflitos. Desse modo, a relação com o pai, que eventualmente poderia oferecer algum tipo de satisfação compensatória para o vínculo tempestuoso mantido com a mãe e a irmã, fica assim despotencializada. O vínculo com o irmão é descrito como afetivamente distante, "como se ele não existisse ali”, e a relação com a irmã é conflituosa, pois Marcela considera que ela "é explosiva como a mãe". Marcela apontou que o relacionamento familiar, antes da ocorrência do TA, era mais tranquilo, e que a origem dos conflitos estava relacionada com o fato de os familiares considerarem seus sintomas "definitivamente uma frescura”. As peculiaridades que envolvem as relações familiares podem ser melhor visualizadas na Figura 1. 


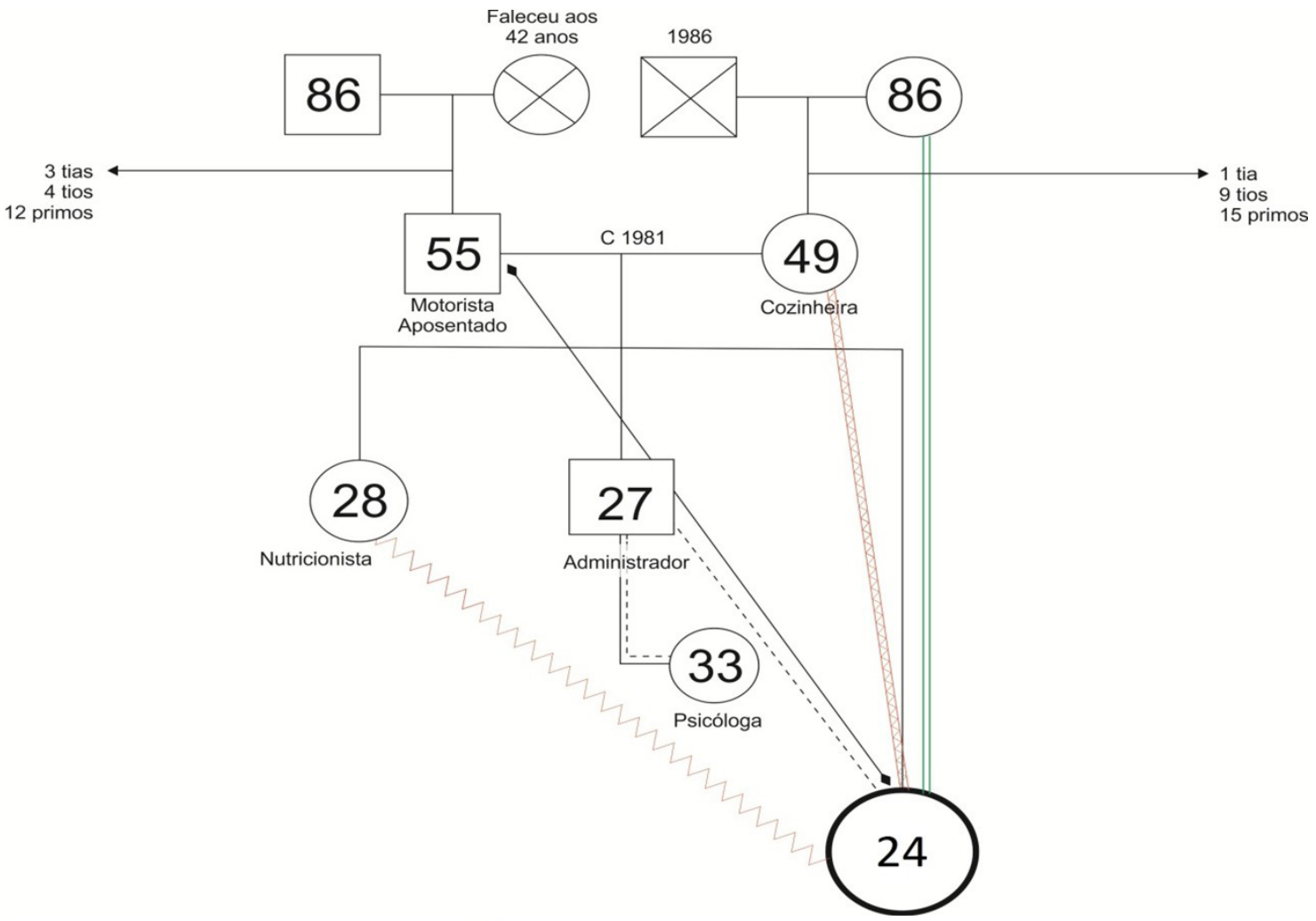

Legenda:

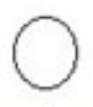

Mulher

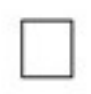

Homem

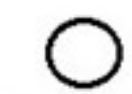

Pessoa indice

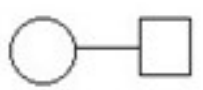

Casamento

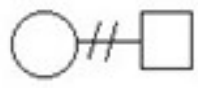

Divórcio

Relacionamento:

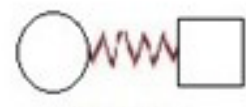

Conflituoso

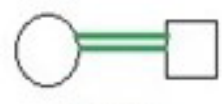

Aliança

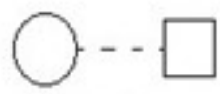

Distante

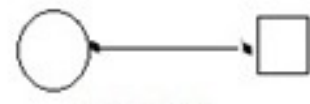

Vulnerável

Figura 1.

Genograma de Marcela.

Os dados refletem uma estrutura familiar na qual a mãe aparece como a principal responsável pelo cuidado e mediação das relações familiares, enquanto o pai ocupa um lugar secundário, mais periférico. Essa configuração do padrão de comunicação familiar não se diferencia da encontrada em famílias com filhos adolescentes sem evidências de diagnóstico psicopatológico (Wagner, Falcke, Silveira, \& Mosmann, 2002). Todavia, no caso investigado neste estudo, nota-se marcada ambivalência na percepção do apoio proveniente da rede familiar. Marcela reconhece que, apesar das cobranças, ameaças e brigas frequentes entre os membros da família, essas pessoas representam sua fonte de apoio mais significativa, juntamente com o namorado. No entanto, a participante mencionou que vivenciava muita insatisfação com o apoio familiar recebido pelo fato deste estar sempre acompanhado de muita exigência e crítica excessiva.

Apesar de tudo, assim, são... são meus amigos, sabe? São pessoas assim... que eu digo que são amigos mesmo, que são pessoas que eu posso contar em qualquer momento, que sempre me escutaram, sabe? 
Não, eu tenho um apoio, sabe? Mas, assim, eu acho que tem horas que é um apoio, assim... não da forma que... como eu gostaria, sabe? Porque imagina você viver toda sua vida sendo cobrada, ameaçada, sabe, assim? Então você fica com medo, você vive sempre com medo, assim... nossa, sempre tendo que atender as expectativas.

Esses excertos de fala de Marcela evidenciam a discrepância existente entre o apoio social recebido e a percepção que ela tem desse apoio, com o qual se mostra claramente insatisfeita. Estudos destacam a rede social familiar como a principal fonte de apoio aos indivíduos afetados por TAs. Em contrapartida, evidencia-se insatisfação com o apoio social recebido por parte da rede familiar, sendo que tal insatisfação pode também estar articulada às características do funcionamento da personalidade das pacientes e às suas manifestações sintomáticas (Leonidas \& Santos, 2013; 2014; 2015b; Leonidas et al., 2013; Limbert, 2010; Peres \& Santos, 2011).

Existem evidências de que as redes familiares de apoio têm impacto positivo sobre os comportamentos saudáveis, influenciando tanto a prevenção quanto o tratamento de problemas, sendo consideradas peças-chave na recuperação (Bullock, 2004). $\mathrm{Na}$ interface do apoio e das redes sociais com a promoção de saúde, essa autora sustenta que tais construtos estão associados à redução das taxas de morbimortalidade, favorecendo a melhora de doenças graves e o aumento da adoção de práticas preventivas de cuidado à saúde. Ao considerar a relação entre apoio social $\mathrm{e}$ saúde, a autora define o conceito de apoio como o fornecimento ou recebimento de assistência, e sugere que a percepção que o indivíduo tem desse apoio pode ser tanto positiva quanto negativa e, por conseguinte, pode afetar a saúde também de forma positiva ou negativa.

Frente ao apoio percebido, Marcela refere viver sob constante pressão devido ao conflito entre seu desejo de emagrecer e as cobranças acentuadas que recebe não apenas dos pais, que exigem que ela não reincida nas condutas de restrição alimentar, como também de pessoas da faculdade que frequenta, já que os professores percebem que ela não está bem e também "cobram" que ela se cuide. Além disso, a insatisfação sentida também está relacionada com a ausência de redes sociais de amigos. Marcela relata que não é aceita nessas redes por não gostar de sair de casa para comer, uma atividade social que colegas da faculdade compartilham com alguma frequência.

Aí nisso, você entra naquele ciclo vicioso, porque diz para eles: "Ai, não como isso, não como aquilo", mas se você não come você também não tem amigos, você não é aceita, porque as pessoas te excluem, de certa forma as pessoas te excluem em tudo. E você também não quer ser colocada naquela posição: "Ai, eu sou diferente, eu sou doente, eu sou isso, eu sou aquilo...".

Se de um lado Marcela sofre por se sentir excluída de seu círculo social, por outro lado também relata um movimento de se autoexcluir de sua rede pessoal significativa, já que, para interagir com os amigos, entende que é preciso comer ou estar perto do alimento, o que lhe suscita aversão e desperta sentimentos disfóricos, como asco, remorso e culpa. Além disso, a participante pondera que o fato de não gostar de estar ao lado de muitas pessoas a impede de ter uma vida social diversificada e minimamente satisfatória. Frequentemente, sente-se observada pelas pessoas, o que aumenta seu desconforto. Acredita que as pessoas sempre ficam observando seus passos e, por isso, evita frequentar eventos sociais, tais como festas e bares, nos quais se sente exposta e vulnerável. O uso abusivo de laxantes também foi considerado um vício que a impedia de manter uma relação próxima e genuína com as amigas por exemplo, inibindo a possibilidade de dormir na casa delas, um hábito comum entre jovens de sua idade.

Acho que, de certa forma, sabe, até eu que acabava me colocando um pouco de lado também, mas aí com o tempo, sabe, assim, é... Acho que o medo de você ser excluída é tão grande que você começa a se inteirar mais, ir junto, fazer as coisas também, só que a sua culpa, seu sentimento de remorso, aquela coisa assim... é muito, muito grande, sabe assim?

Em contraposição a essa percepção, o desejo de ser incluída nas redes sociais de amigos foi um tema recorrente nos relatos da participante. Referiu fazer uso de algumas táticas para "se inteirar"(socializar-se), tais como comer apenas frutas em casa para conseguir ingerir minimamente outros alimentos (por exemplo, chocolate) junto aos amigos na faculdade. Essa estratégia parecia funcionar como fator de redução da ansiedade e dos sentimentos negativos que 
experimentava; ao mesmo tempo, servia para aproximá-la dos amigos. No entanto, a participante sente que algumas amigas se aproximavam dela apenas "por interesse", pois ela costuma fazer com esmero todos os trabalhos da faculdade. Ela é admirada por essa habilidade, porém as amigas não a incluem em outras atividades sociais. Essa percepção faz com que Marcela se sinta ainda mais excluída da sua rede de amigos. O sentimento de não pertencimento intensifica suas vivências de desamparo e menos-valia. A ambivalência também está presente entre o desejo de integrar-se à rede e a necessidade defensiva de fortificar suas fronteiras psíquicas e se isolar por completo do mundo, de modo a evitar a frustração.

Ah, sabe assim, pô, é chato, sabe? Tá todo mundo junto, assim... Você quer ficar sozinha? Você não quer ficar sozinha, sabe. Daí... às vezes, eu até dava umas indiretas, assim. Mas eu não tenho coragem de chegar e falar.

A interação com as pessoas da rede social significativa parece exercer influência em várias dimensões do quadro sintomatológico de AN. Marcela atribui o início dos sintomas psicopatológicos à perda de pessoas próximas, às comparações que ela própria fazia com sua mãe e irmã (que, segundo ela, eram muito magras), aos apelidos depreciativos que a irmã lhe atribuía, ao rígido controle alimentar exercido pela mãe e ao fato de o menino que ela gostava na infância começar a namorar uma adolescente magra. Segundo seu relato, esses fatores resultaram em uma autoestima marcadamente rebaixada, sentimentos de inferioridade e desejo de "provar aos outros que eu podia". A combinação desses elementos, no seu entendimento, a teria levado a adotar hábitos radicais de restrição alimentar.

Marcela contou que namorava havia dois anos e meio, e que mantinha com o namorado um relacionamento que considerava saudável. Ela o descreveu como alguém paciente e com quem ela compartilhava muitas afinidades, o que assegurava um sentimento de segurança íntima e cumplicidade. Relatou também que o namorado havia sido uma fonte de apoio significativa em vários momentos difíceis pelos quais ela passara desde que se mudou de sua cidade de origem para cursar faculdade. A estabilidade desse relacionamento afetivo e o nível de satisfação auferido com o vínculo são indicadores de recursos valiosos no contexto existencial de Marcela.
Antes da ocorrência do TA, Marcela considerava que tinha uma ampla rede social de amigos, uma vez que "dava mais abertura" para as pessoas de seu ambiente social e "era mais sociável". Atualmente, "tem sempre um pé atrás" e "se fechou muito" para as pessoas. Esse afastamento acabou por restringir o tamanho e a composição de sua rede social significativa e por impedir a realização de atividades de lazer, até que o vínculo com o namorado passou a ser a única companhia social e a fonte mais significativa de apoio para a participante. Juntos, frequentam alguns bares e passeiam no shopping.

Como é possível visualizar no Mapa de Rede (Figura 2), a rede social significativa de Marcela era compostapor, aproximadamente, novemembros, distribuídos entre os quadrantes da Família, do Serviço de Saúde (Grata) e da Faculdade, configurando uma rede social de tamanho médio (Orlandi, 2011). Vale ressaltar que a participante inseriu a orientadora e as "amigas da faculdade" no quadrante reservado ao Trabalho e Faculdade, sem atribuir uma quantidade específica para o número de amigas, o que dificultou a quantificação do número de membros de sua rede. Não foram inseridas pessoas no quadrante de Amigos nem no da Comunidade. A maioria dos membros da rede $(n=6)$ concentrava-se no quadrante da Família, na parte de Relações Íntimas, sendo eles: mãe, pai, avó, primo, prima e namorado. A orientadora, as amigas da faculdade e a psiquiatra do Serviço de Saúde foram consideradas membros integrantes das Relações Sociais. Apenas uma paciente do serviço, com quem Marcela mantinha contato esporádico, foi considerada Relação Distante. Os membros de cada quadrante mantinham conexões entre si, e a família também estava conectada com a profissional do serviço de saúde. Assim, considera-se que a rede social de Marcela apresenta baixa "densidade", definida como o grau de conexões que os membros da rede mantêm entre si (Sluzki, 1997).

A Figura 2 evidencia que há escassez de membros da rede para além do quadrante da Família, e que as relações com os membros dos outros quadrantes que configuram o Mapa de Rede podem ser consideradas superficiais, uma vez que são pessoas com quem a participante mantém contato frequente, porém destituído de intimidade. Nesse sentido, pode-se considerar que o potencial de apoio social proveniente das redes de Amigos, Faculdade e Comunidade encontra-se seriamente prejudicado. 


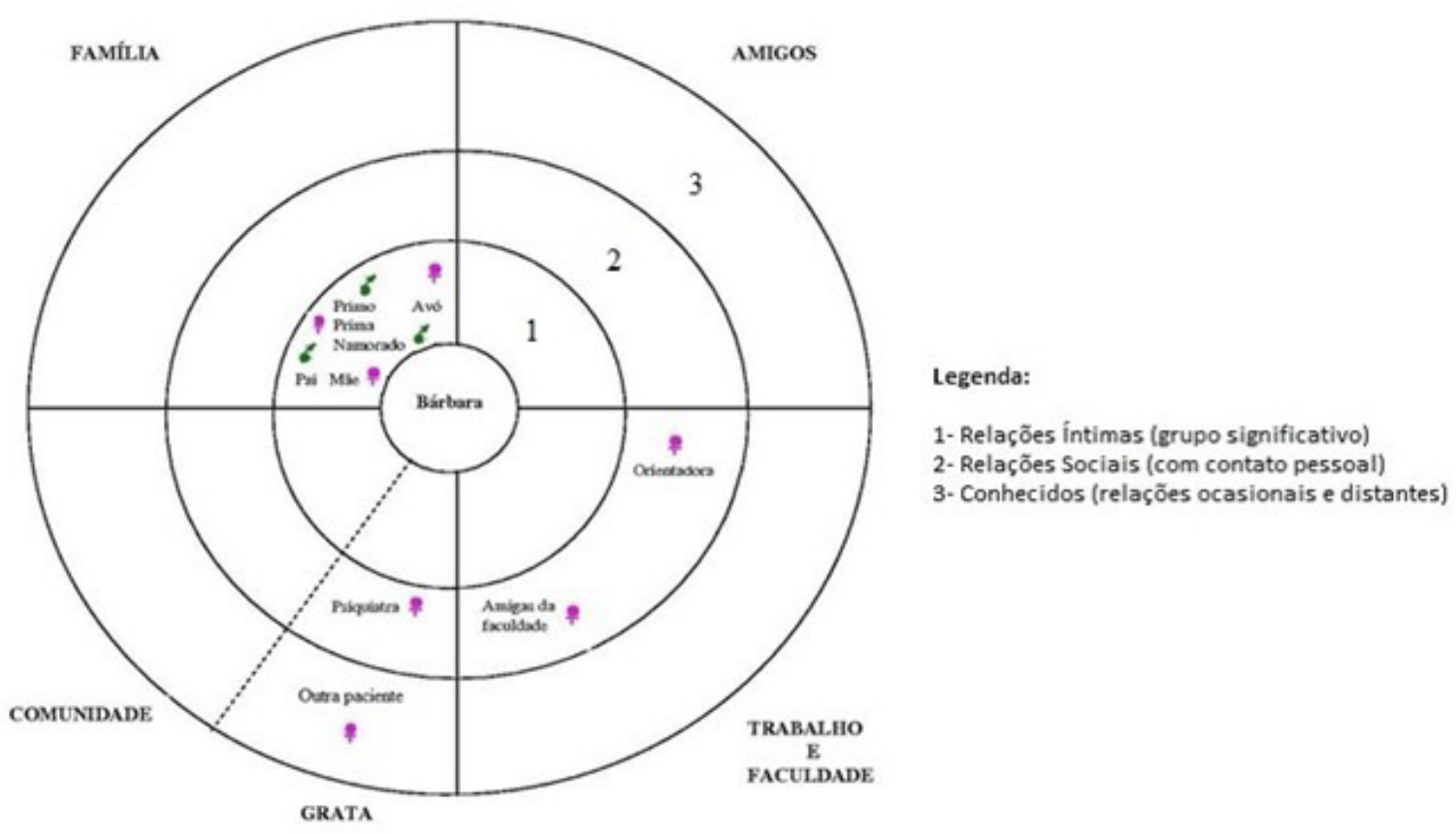

Figura 2.

Mapa de Rede de Marcela.

Face aos resultados obtidos, é possível afirmar que os achados estão em conformidade com outras pesquisas publicadas na área, que sugerem a relevância dos fatores relacionados à organização e funcionamento familiar na sustentação da sintomatologia dos TAs (Leonidas \& Santos, 2013; 2015a; 2015b; 2017; Leonidas, Crepaldi, \& Santos, 2013; Siqueira et al., 2020). Por outro lado, o presente estudo avança no conhecimento já consolidado ao mostrar, com as nuances e minúcias que um estudo de caso pode oferecer, a influência marcante da rede familiar sobre o funcionamento psicodinâmico da participante e, consequentemente, sobre a precipitação e manutenção do quadro clínico.

Além disso, os padrões de relacionamento identificados na família de Marcela também corroboram evidências disponíveis na literatura científica que sugerem a existência de um vínculo fusional e conflituoso da filha com a mãe e uma relação emocionalmente distante com o pai (Leonidas \& Santos, 2013; 2015a; 2015b; 2017; Leonidas, Crepaldi, \& Santos, 2013). Essa configuração vincular entre pais e filhas parece acentuar a sensação de incompreensão e ausência de proteção frequentemente relatada por jovens acometidas por TAs, o que é congruente com a ideia de que melhorar a adaptabilidade, coesão e comunicação familiar são dimensões importantes no tratamento (Laghi et al., 2017).

Nesse sentido, os resultados sistematizados por este estudo de caso reforçam a necessidade de inclusão da família no tratamento, mas ao mesmo tempo dão pistas no sentido de que essa incorporação dos cuidadores familiares não pode se restringir a uma dimensão meramente informativa ou psicoeducativa, a exemplo do que acontece com os programas de educação em saúde ou orientação de pais. Os familiares necessitam contar com um espaço próprio de cuidado terapêutico, no qual possam se apropriar de suas questões subjetivas e interacionais, e reconhecer as conexões entre a sintomatologia das filhas e o modo de se constituírem os vínculos familiares.

Como a terapia familiar é um recurso dispendioso e nem sempre acessível, acaba por se tornar inexequível na realidade da maioria das famílias assistidas em nosso contexto. Desse modo, recomenda-se uma abordagem fundamentada na oferta de intervenções multifamiliares, como os grupos operativos com finalidade terapêutica, que contribuem para que os pais possam se sentir amparados no processo de reconstrução de vínculos afetivos mais genuínos e solidários, fortalecendo sua disponibilidade para fornecer apoio e continência 
às necessidades psíquicas de suas filhas (Santos, Leonidas, \& Costa, 2016; Siqueira et al., 2020; Souza \& Santos, 2009). Estratégias como essa, integradas em um plano de tratamento de base familiar, podem contribuir para promover o bem-estar e satisfação familiar, favorecendo a melhora do quadro psicopatológico do membro acometido (Trainor et al., 2020).

\section{Considerações Finais}

O presente estudo teve como objetivo investigar a configuração da rede social significativa de uma mulher com AN, buscando articular a percepção do apoio social recebido e o curso do transtorno. Resguardados os limites da investigação, foi possível elucidar algumas relações entre a forma como a participante percebe o apoio social recebido e a dinâmica do TA. Partindo-se do conhecimento científico já consolidado de que quanto mais positiva for a percepção de apoio recebido, melhores são as condições de um indivíduo se recuperar (ou se adaptar social e funcionalmente) de um transtorno mental, este estudo apresenta resultados relevantes para estimar se o apoio social se mostra satisfatório ou não para a paciente em questão, e se exerce influência sobre a frequência e gravidade dos sintomas apresentados.

Observou-se que a rede social significativa da participante tem tamanho médio, com membros mal distribuídos entre os quadrantes do Mapa de Rede, havendo maior concentração na esfera da Família. No que concerne à dinâmica familiar, nota-se um distanciamento emocional entre a jovem e os membros da família e a existência de um relacionamento fusional e conflituoso com a mãe, a quem a participante caracterizou como pessoa "explosiva" e pouco confiável. A incompreensão da família acerca do TA parece despertar sentimentos de desamparo e exclusão, fazendo com que Marcela se sinta permanentemente cobrada e ameaçada. Além disso, a paciente se sente na obrigação de responder às exigências dos pais, ao mesmo tempo em que se sente incompetente para atender essas expectativas, o que contribui para reduzir sua autoestima e acentuar sua sensação de desamparo.

Não obstante os problemas enfrentados nos relacionamentos, a rede familiar, incluindo o namorado, foi caracterizada pela participante como sua fonte mais significativa de apoio social. Por conseguinte, há ambivalência em relação a esse apoio, pois apesar de ser o mais significativo, é também percebido como insatisfatório e fonte de sofrimento.

Vale destacar alguns aspectos do funcionamento da personalidade da participante, como insegurança e desconfiança vivenciadas nos relacionamentos interpessoais, condutas defensivas de evitação e busca de isolamento, que resultavam em afastamento concreto das pessoas de seu contexto de vida, intensificando ainda mais os sentimentos de solidão e desamparo e, consequentemente, restringindo ainda mais o tamanho da rede pessoal e seu potencial de apoio.

Com base nessas considerações, espera-se que este estudo contribua para o conhecimento da configuração dos vínculos e das redes de apoio social no contexto da psicopatologia do comportamento alimentar. Entende-se que esse conhecimento é valioso para o profissional de saúde na medida em que ele souber instrumentalizá-lo em sua prática clínica, permitindo fortalecer as redes de apoio como estratégias facilitadoras e ampliadoras das ações de assistência em saúde. Novos estudos nessa área podem favorecer o aprimoramento do tratamento dos TAs, uma vez que as relações entre apoio social e indicadores de saúde/ doença têm sido cada vez mais abordadas e comprovadas pela literatura científica.

\section{Referências}

American Psychiatric Association. (2013). Diagnostic and statistical manual of mental disorders: DSM-5 (5a ed.). Washington, D. C.: American Psychiatric Association. https://doi.org/10.1176/appi.books.9780890425596

Andrade, T. F., \& Santos, M. A. (2009). A experiência corporal de um adolescente com transtorno alimentar. Revista Latino-Americana de Psicopatologia Fundamental, 12(3), 454-468.

Attili, G., Di Pentima, L., Toni, A., \& Roazzi, A. (2018). High anxiety attachment in eating disorders: Intergenerational transmission by mothers and fathers. Paidéia (Ribeirão Preto), 28. http://dx.doi.org/10.1590/1982-4327e2813

Brusset, B. (2003). Introdução geral. In B. Brusset, A. Couvreur, \& A. Fine (Orgs.), A bulimia (pp. 7-13). São Paulo, SP: Escuta.

Bullock, K. (2004). Family social support. In P. J. Bomar (Ed.), Promoting health in families: Applying research and theory to nursing practice (pp. 141-161). Philadelphia, PA: Saunders. 
Costa, L. R. S., \& Santos, M. A. (2016). Cuidado paterno e relações familiares no enfrentamento da anorexia e bulimia. In D. Bartholomeu, J. M. Montiel, A. A. Machado, A. R. Gomes, G. Couto, \&V. Cassep-Borges (Orgs.), Relações interpessoais: Concepções e contextos de intervenção e avaliação (pp. 253-279). São Paulo, SP: Vetor.

Eddy, K. T., Tabri, N., Thomas, J. J., Murray, H. B., Keshaviah, A., Hastings, E., Edkins, K., Krishna, M., Herzog, D. B., Keel, P. K., \& Franko, D. L. (2017). Recovery from anorexia nervosa and bulimia nervosa at 22-year follow-up. Journal of Clinical Psychiatry, 78(2), 184-189. https:// doi.org/10.4088/JCP.15m10393

Godoy, A. S. (2006). Estudo de caso qualitativo. In C. K. Godoi, R. Bandeira-de-Mello, \& A. B. Silva (Orgs.), A pesquisa qualitativa em estudos organizacionais: Paradigmas, estratégias e métodos (pp. 115-146). São Paulo, SP: Saraiva.

Goulart, D. M., \& Santos, M. A. (2012). Corpo e palavra: Grupo terapêutico para pessoas com transtornos alimentares. Psicologia em Estudo (Maringá), 17(4), 607-617.

Goulart, D. M., \& Santos, M. A. (2015). Psicoterapia individual em um caso grave de anorexia nervosa: A construção da narrativa clínica. Psicologia Clínica, 27(2), 201-227.

King, V., Gerisch, B., \& Schreiber, J. (2020). “...To really have everything completely perfect”: On the psychodynamics of contemporary forms of body optimization. Psychoanalytic Psychology, 37(2), 148-157. https://doi.org/10.1037/pap0000287

Kreling, D. B., \& Santos, M. A. (2005). Anorexia nervosa: Um relato de atendimento clínico realizado em contexto multidisciplinar. In M. A. Santos, C. P. Simon, \& L. L. Melo-Silva (Orgs.), Formação em Psicologia:Processos clínicos (pp. 143-161). São Paulo, SP: Vetor.

Laghi, F., Pompili, S., Zanna, V., Castiglioni, M. C., Criscuolo, M., Chianello, I., Mazzoni, S., \& Baiocco, R. (2017). How adolescents with anorexia nervosa and their parents perceive family functioning? Journal of Health Psychology, 22(2), 197-207. https://doi.org/10.1177/1359105315597055

Lavall, E., Olschowsky, A., \& Kantorski, L. P. (2009). Avaliação de família: Rede de apoio social na atenção em saúde mental. Revista Gaúcha de Enfermagem, 30(2), 198-205.

Leonidas, C. (2012). Redes sociais e apoio social no contexto dos transtornos alimentares. (Dissertação de mestrado), Universidade de São Paulo, Ribeirão Preto, SP, Brasil.

Leonidas, C., \&Santos, M.A. (2012). Imagem corporal ehábitos alimentares na anorexia nervosa: Uma revisão integrativa da literatura. Psicologia:Reflexão e Crítica, 25(3), 550-558. https://doi.org/10.1590/S0102-79722012000300015

Leonidas, C., \& Santos, M. A. (2013). Redes sociais significativas de mulheres com transtornos alimentares. Psicologia: Reflexão e Crítica, 26(3), 561-571. https://doi.org/10.1590/S0102-79722013000300016

Leonidas, C., \& Santos, M. A. (2014). Social support networks and eating disorders: An integrative review of the literature. Neuropsychiatric Disease and Treatment, 10, 915-927. https://doi.org/10.2147/NDT.S60735

Leonidas, C., \& Santos, M. A. (2015a). Relacionamentos afetivo-familiares em mulheres com anorexia e bulimia. Psicologia: Teoria e Pesquisa, 31(2), 181-191. https://doi.org/10.1590/0102-37722015021711181191

Leonidas,C.,\&Santos,M.A.(2015b).Relaçõesfamiliaresnostranstornosalimentares:OGenogramacomoinstrumento de investigação. Ciência \& Saúde Coletiva, 20(5), 1435-1447.https:// doi.org/10.1590/1413-81232015205.07802014

Leonidas, C., \& Santos, M. A. (2017). Emotional meanings assigned to eating disorders: Narratives of women with anorexia and bulimia nervosa. Universitas Psychologica, 16(4), 1-13. https://doi.org/10.11144/Javeriana. upsy16-4.emae

Leonidas, C., \& Santos, M. A. (2020). Symbiotic illusion and female identity construction in eating disorders: A psychoanalytical psychosomatics’ perspective. Ágora: Estudos em Teoria Psicanalítica, 23(1), 84-93. https://doi.org/10.1590/1809-44142020001010

Leonidas, C., Crepaldi, M. A., \& Santos, M. A. (2013). Bulimia nervosa: Uma articulação entre aspectos emocionais e rede de apoio social. Psicologia: Teoria e Prática, 15(2), 62-75.

Leonidas, C., Nazar, B. P., Munguía, L., \& Santos, M. A. (2019). How do we target the factors that maintain anorexia nervosa? A behaviour change taxonomical analysis. International Review of Psychiatry, 31(4), 403-410. https://doi.org/10.1080/09540261.2019.1624509

Limbert, C. (2010). Perceptions of social support and eating disorder characteristics. Health Care for Women International, 31(2), 170-178. https://doi.org/10.1080/07399330902893846

Moré, C. L. O. O. (2005). As redes pessoais significativas como instrumento de intervenção psicológica no contexto comunitário. Paidéia (Ribeirão Preto), 15(31), 287-297. https://doi.org/10.1590/S0103-863X2005000200016 
Moré, C. L. O. O., \& Crepaldi, M. A. (2012). O mapa de rede social significativa como instrumento de investigação no contexto da pesquisa qualitativa. Nova Perspectiva Sistêmica, 21(43), 84-98.

Oliveira-Cardoso, E. A., \& Santos, M. A. (2012). Avaliação psicológica de pacientes com anorexia e bulimia nervosas: Indicadores do método de Rorschach. Fractal: Revista de Psicologia, 24(1), 159-174. https://doi.org/10.1590/ S1984-02922012000100011

Oliveira-Cardoso, E. A., Valdanha-Ornelas, E. D., Leonidas, C., Pessa, R. P., Santos, J. E., \& Santos, M. A. (2018). Assistência em transtornos alimentares como parte do itinerário formativo do aluno de Psicologia: Aprendizado em equipe interdisciplinar. In L. C. S. Elias, C. M. Corradi-Webster, E. A. Oliveira-Cardoso, S. D. Barreira, \& M. A. Santos (Orgs.), Formação profissional em psicologia: Práticas comprometidas com a comunidade (pp. 82-108). Ribeirão Preto, SP: Sociedade Brasileira de Psicologia.

Oliveira-Cardoso, E. A., Von Zuben, B. V., \& Santos, M. A. (2014). Quality of life of patients with anorexia and bulimia nervosa. Demetra:Food, Nutrition and Health, 9(1. supl.), 329-340. https://doi.org/10.12957/demetra.2014.10346

Orlandi, R. (2011). Participação da rede social significativa de mulheres que vivem e convivem com o HIV no enfrentamento da soropositividade (Tese de doutorado). Universidade Federal de Santa Catarina, Florianópolis, SC.

Peres, R. S., \& Santos, M. A. (2005). Considerações gerais e orientações práticas acerca do emprego de estudos de caso na pesquisa científica em Psicologia. Interações: Estudos e Pesquisa em Psicologia, 10(20), 109-126.

Peres, R. S., \& Santos, M. A. (2011). Técnicas projetivas na avaliação de aspectos psicopatológicos da anorexia e bulimia. PsicoUSF, 16(2), 185-192. https://doi.org/10.1590/S1413-82712011000200007

Rosa, B. P., \& Santos, M. A. (2011). Comorbidade entre bulimia e transtorno de personalidade borderline: Implicações para o tratamento. Revista Latinoamericana de Psicopatologia Fundamental, 14(2), 268-282. https:// doi.org/10.1590/S141547142011000200005

Santos, M. A., Leonidas, C., \& Costa, L. R. S. (2016). Grupo multifamiliar no contexto dos transtornos alimentares: A experiência compartilhada. Arquivos Brasileiros de Psicologia, 68(3), 43-58.

Siqueira, A. B. R., Santos, M. A., \& Leonidas, C. (2020). Confluências das relações familiares e transtornos alimentares: Revisão integrativa da literatura. Psicologia Clínica, 32(1), 123-149. http://dx.doi.org/10.33208/ PC1980-5438v0032n01A06

Sluzki, C. E. (1997). A rede social na prática sistêmica: Alternativas terapêuticas (C. Berliner, Trad.). São Paulo, SP: Casa do Psicólogo.

Smink, F. R. E., Hoeken, D. V., \& Hoek, H. W. (2013). Epidemiology, course, and outcome of eating disorders. Current Opinion, 26(6), 543-548. https://doi.org/10.1097/YCO.0b013e328365a24f

Souza, A. P. L., Valdanha-Ornelas, É. D., Santos, M. A., \& Pessa, R. P. (2019). Significados do abandono do tratamento para pacientes com transtornos alimentares. Psicologia: Ciência e Profissão, 39, 1-16. https://doi. org/10.1590/1982-3703003188749

Souza, L. V., \& Santos, M. A. (2009). A construção social de um grupo multifamiliar no tratamento dos transtornos alimentares. Psicologia: Reflexão e Crítica, 22(3), 483-492. http://dx.doi.org/10.1590/S0102-79722009000300020

Stake, R. E. (2005). Qualitative case studies. In N. K. Denzin \& Y. S. Lincoln (Eds.), The Sage handbook of qualitative research (pp. 443-466). Thousand Oaks: Sage.

Trainor, C., Gorrell, S., Hughes, E. K., Sawyer, S. M., Burton, C., \& Le Grange, D. (2020). Family-based treatment for adolescent anorexia nervosa: What happens to rates of comorbid diagnoses? European Eating Disorder Review, 28(3), 1-7. https://doi.org/10.1002/erv.2725

Valdanha, E. D., Scorsolini-Comin, F., \& Santos, M. A. (2013). Anorexia nervosa e transmissão psíquica transgeracional. Revista Latinoamericana de Psicopatologia Fundamental, 16(1), 71-88. https://doi.org/10.1590/ S1415-47142013000100006

Wagner, A., Falcke, D., Silveira, L. M. B. O., \& Mosmann, C. P. (2002). A comunicação em famílias com filhos adolescentes. Psicologia em Estudo (Maringá), 7(1), 75-80. https://dx.doi.org/10.1590/S1413-73722002000100010

Wendt, N. C., \& Crepaldi, M. A. (2008). A utilização do Genograma como instrumento de coleta de dados na pesquisa qualitativa. Psicologia: Reflexão e Crítica, 21(2), 302-310. https://doi.org/10.1590/S0102-79722008000200016

Yin, R. K. (2005) Estudo de caso: Planejamento e método (D. Grassi, Trad., 3a ed.). Porto Alegre, RS: Artmed. 


\section{Carolina Leonidas}

Instituto de Educação, Letras, Artes, Ciências Humanas e Sociais, Universidade Federal do Triângulo Mineiro, Uberaba-MG. Brasil.

E-mail: carol.leonidas@gmail.com

(D) https://orcid.org/0000-0002-6558-3943

Manoel Antônio dos Santos

Departamento de Psicologia, Faculdade de Filosofia, Ciências e Letras de Ribeirão Preto, Universidade de São Paulo, São Paulo - SP. Brasil.

E-mail: masantos@ffclrp.usp.br

(D) http://orcid.org/0000-0001-8214-7767

Endereço para envio de correspondência:

Instituto de Educação, Letras, Artes, Ciências Humanas e Sociais, Universidade Federal do Triângulo Mineiro. Rua Vigário Carlos, Nossa Senhora da Abadia. CEP: 38025-460. Uberaba - MG. Brasil.

Recebido $12 / 06 / 2018$

Aceito 07/02/2020

Received 12/06/2018

Approved 07/02/2020

Recibido 12/06/2018

Aceptado 07/02/2020

Como citar: Leonidas, C., \& Santos, M. A. (2020). Percepção do Apoio Social e Configuração Sintomática na Anorexia Nervosa. Psicologia: Ciência e Profissão, 40, 1-14. https://doi.org/10.1590/1982-3703003207693

How to cite: Leonidas, C., \& Santos, M. A. (2020). Perception of Social Support and Symptomatic Configuration in Anorexia Nervosa. Psicologia: Ciência e Profissão, 40, 1-14. https://doi.org/10.1590/1982-3703003207693

Cómo citar: Leonidas, C., \& Santos, M. A. (2020). Percepción del Apoyo Social y la Configuración Cintomática en la Anorexia Nerviosa. Psicologia: Ciência e Profissão, 40, 1-14. https://doi.org/10.1590/1982-3703003207693 